\title{
Correction to: Minimally invasive robotic-assisted combined colorectal and liver excision surgery: feasibility, safety and surgical technique in a pilot series
}

\author{
Graziano Ceccarelli ${ }^{1,2} \cdot$ Aldo Rocca $^{2,3} \cdot$ Michele De Rosa $^{1} \cdot$ Andrea Fontani $^{2} \cdot$ Fabio Ermili $^{1}$ - Enrico Andolfi ${ }^{2}$. \\ Walter Bugiantella ${ }^{1}$. Giovanni Battista Levi Sandri ${ }^{4}[$
}

Published online: 10 May 2021

(C) Italian Society of Surgery (SIC) 2021

Correction to: Updates in Surgery https://doi.org/10.1007/s13304-021-01009-3

After publication of the article we found several mistakes in the figures and tables. The corrected Figs. 1, 2, 3 and Tables 1, 2, 3, 4, 5 are given below.

The original article has been updated.

Publisher's Note Springer Nature remains neutral with regard to jurisdictional claims in published maps and institutional affiliations.

The original article can be found online at https://doi.org/10.1007/ s13304-021-01009-3.

Graziano Ceccarelli

grazianoceccarelli1967@gmail.com

1 General and Robotic Surgery Department, San Giovanni Battista Hospital, Foligno, Perugia, Italy

2 San Donato Hospital, General and Robotic Surgery Unit, Arezzo, Italy

3 Department of Medicine and Health Sciences "V. Tiberio", University of Molise, Via Francesco de Sanctis, 1, 86100 Campobasso, Italy

4 Division of General Surgery and Liver Transplantation, Polo Ospedaliero Interaziendale Trapianti (POIT), San Camillo-Forlanini Hospital, Rome, Italy 


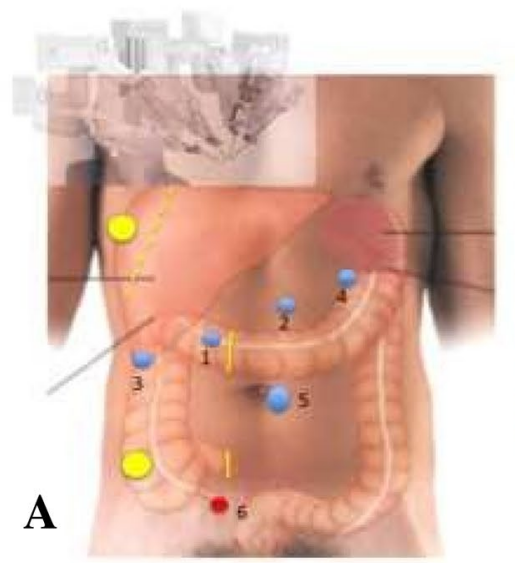

Scenario 1 ; single doking Right liver / Right colectamy

(-) 1-Hobotic camest

- 2/3. Operative nothitic ams

- 4-mohotic netractioe

(2) 5-13nmi suihary part

- Si-funther pait for it colvetomy

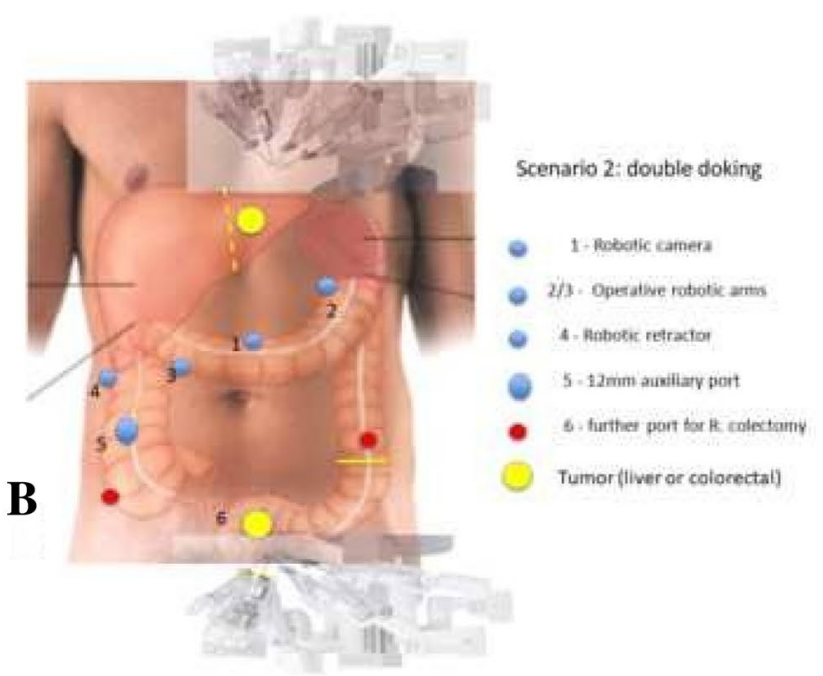

Fig. 1 The docking and trocar placement of the two most frequent scenarios. a Right colon and liver resection, b left sided/rectal colon cancer and liver resection
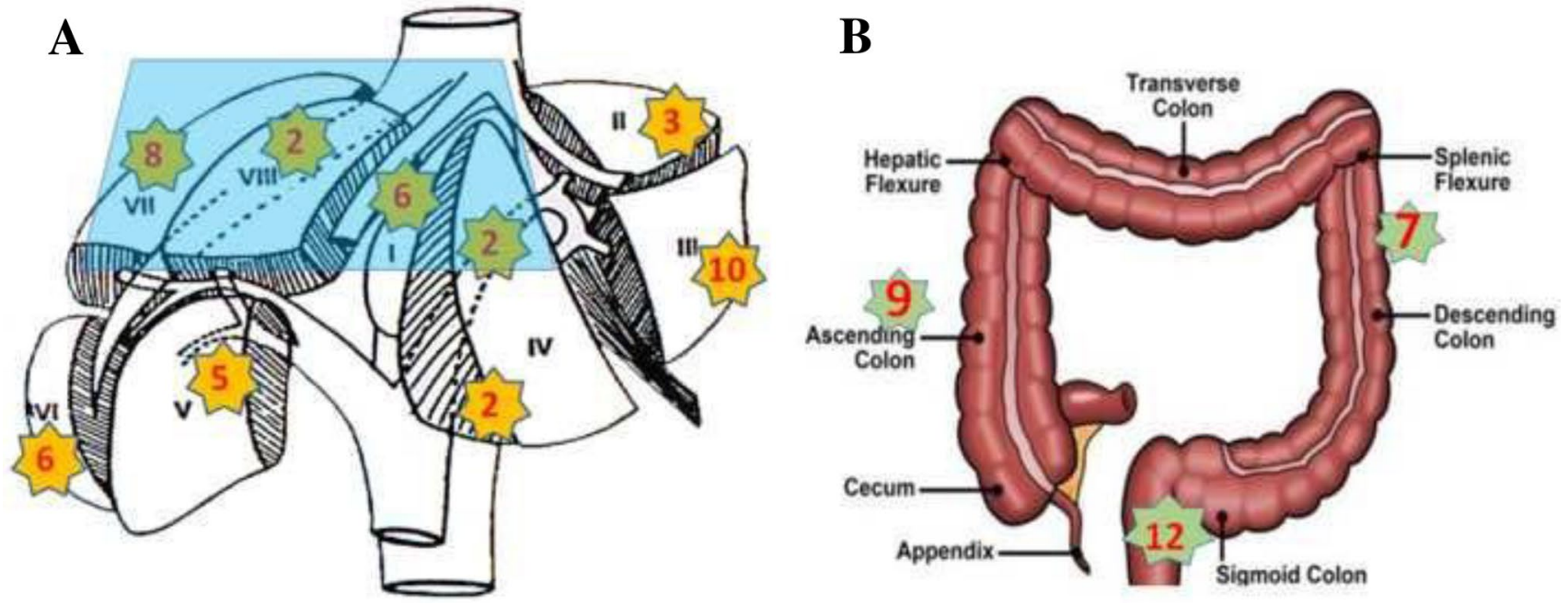

Fig. 2 a Anatomical location of liver nodules resected. Segment IV is represented as IVa and IVb. The Nodules in the unfavourable segments liver are highlighted. b Colorectal tumor distribution 


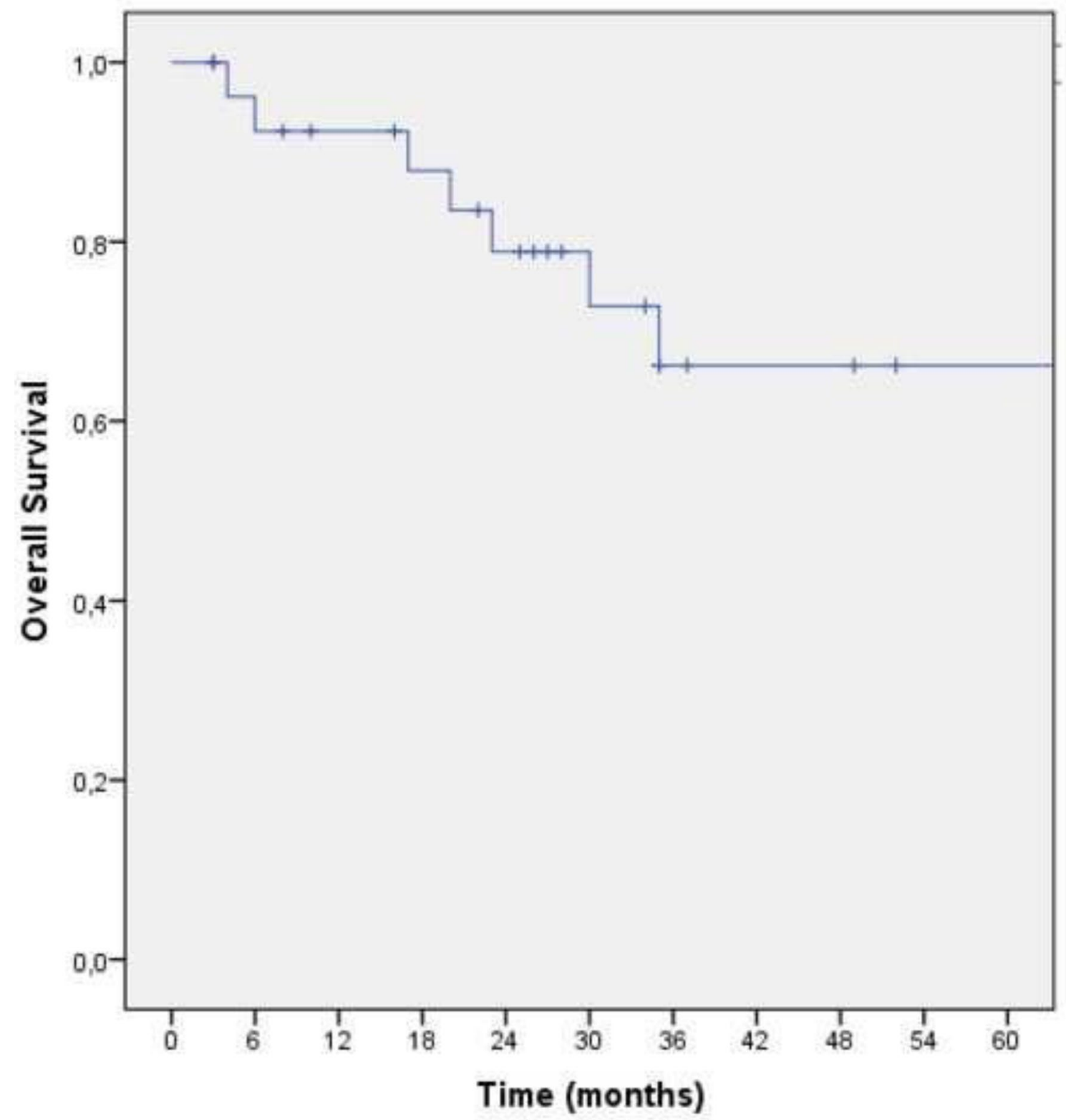

Fig. 3 Kaplan-Meir curve of the overall survival 
Table 1 Patients characteristics

\begin{tabular}{ll}
\hline Gender & \\
Male & 18 \\
Female & 10 \\
Age & $65[54-73]$ \\
BMI & $27.5[15-43]$ \\
ASA & $2(1-3)$ \\
Type of liver resection & \\
Wedge & 20 \\
Segmenctectomy & 5 \\
Left lateral sectionectomy & 1 \\
Right bi segmentectomy & 1 \\
Left hepatectomy & 1 \\
Type of colon resection & \\
Right hemicolectomy & 9 (1 laparoscopic) \\
Left hemicolectomy & 7 (2 laparoscopic) \\
Rectal anterior resection & 10 \\
Hartmann procedure & 1 (laparoscopic) \\
MILES procedure & 1 \\
Specimen extraction site & \\
Pfannenstiel & 17 \\
Ombelical trocar & 7 \\
Suprapubic mini-laparotomy & 4 \\
\hline
\end{tabular}

Data are expressed in median, IQR
Table 2 Perioperative data according to Clavien-Dindo classification

\begin{tabular}{llll}
\hline Age & BMI & ASA & CLAVIEN \\
\hline 75 & 24.91 & 1 & 1 \\
31 & 19.59 & 1 & $3 \mathrm{~b}$ \\
51 & 31.31 & 1 & 1 \\
39 & 29.11 & 1 & 1 \\
53 & 40.83 & 1 & 1 \\
39 & 21.60 & 1 & $3 \mathrm{~b}$ \\
66 & 23.62 & 2 & 1 \\
83 & 26.60 & 2 & 1 \\
70 & 29.4 & 2 & 1 \\
55 & 27.73 & 2 & 2 \\
54 & 23.12 & 2 & 1 \\
85 & 25.00 & 2 & 1 \\
77 & 29 & 2 & 2 \\
64 & 24.00 & 2 & 1 \\
68 & 30.38 & 2 & 1 \\
63 & 27.77 & 2 & 1 \\
70 & 25.6 & 2 & 1 \\
59 & 26.70 & 2 & 1 \\
63 & 27.40 & 2 & 1 \\
63 & 31.00 & 3 & $3 \mathrm{a}$ \\
80 & 27.50 & 3 & 1 \\
63 & 31.50 & 3 & 1 \\
80 & 25.60 & 3 & 1 \\
69 & 43.00 & 3 & 2 \\
56 & 29.00 & 3 & 1 \\
53 & 15.77 & 3 & 1 \\
77 & 28.65 & 3 & $1-2$ \\
66 & 25.24 & 3 & $4 \mathrm{~b}$ \\
72 & 23.52 & 3 & 2 \\
\hline & & & \\
57
\end{tabular}

Table 3 Operative data are expressed in median and IQR

\begin{tabular}{ll}
\hline Operation time (min) & $332[280-385]$ \\
Blood loss (cc) & $143[50-600]$ \\
Pringle manouvre & $9 / 28$ \\
Conversion & 2 \\
Complication grade according to Clavien-Dindo classification \\
I-II & 24 \\
IIIa & 1 \\
IIIb & 2 \\
IV & 1 \\
Mortality & 0 \\
Length of stay (days) & $8[7-13]$ \\
\hline
\end{tabular}


Table 4 Tumor characteristics

\begin{tabular}{ll}
\hline $\begin{array}{l}\text { Nodule diameter } \\
\text { Radicality }\end{array}$ & 24.8 [7-66] \\
R0 & 27 \\
R1 & 1 \\
TNM & \\
T1 & 1 \\
T2 & 1 \\
T3 & 24 \\
T4 & 1 \\
\hline
\end{tabular}

Nodule diameter is expressed in $\mathrm{mm}$

Table 5 Anterior rectal resections details

\begin{tabular}{|c|c|c|c|c|}
\hline Primary resection & Liver resection & Pathological finding & TNM & Duration \\
\hline Anterior rectal resection + adesiolysis & Wedge resection VIIS & Upper rectal adenocarcinoma & PT3N1MXG2 & 310 \\
\hline Anterior rectal resection & Wedge resection III & Upper rectal adenocarcinoma & T3 N1B M1 G3 & $270^{\prime}$ \\
\hline Anterior rectal resection & Wedge resection IVA, V, VI, VIIS & Upper rectal adenocarcinoma & T3N2M1 G3 & $540^{\prime}$ \\
\hline Anterior rectal resection & Wedge resection III & Rectal adenocarcinoma & TXNOMX & $420^{\prime}$ \\
\hline Anterior rectal resection & Wedge resection VI-VII & Rectal adenocarcinoma & T3N2M1G3 & $420^{\prime}$ \\
\hline Miles resection + colecistectomy & Wedge resection VI+ III & Rectal adenocarcinoma & PT3N2AM1 G2 & 310 \\
\hline Anterior rectal resection & Wedge resection IV & Rectal adenocarcinoma & T4BN0M1 G3 & $335^{\prime}$ \\
\hline Laparoscopic Hartmann resection & Wedge III & Rectal adenocarcinoma & T3N1BM1A G2 & $285^{\prime}$ \\
\hline Anterior rectal resection + colecistectomy & Wedge resection I, VI, IV B & Rectal adenocarcinoma & T3N1BM1A G3 & $345^{\prime}$ \\
\hline Anterior rectal resection + colecistectomy & Segmentectomy VI & Rectal adenocarcinoma & PT3N2BM1 G2-3 & 380 \\
\hline Anterior rectal resection + colecistectomy & Subsegmentectomy VS & Rectal adenocarcinoma & PT3N1AM1G2 & 370 \\
\hline Anterior rectal resection & Wedge resection IIIS + VS & Rectal adenocarcinoma & T3N2M1 G3 & 400 \\
\hline
\end{tabular}

